\title{
Outcomes of Bariatric Surgery in Morbidly Obese Patients with Multiple Sclerosis
}

\author{
Kalman Bencsath, ${ }^{1,2}$ Adham Jammoul, ${ }^{3}$ Ali Aminian, ${ }^{1,2}$ Hideharu Shimizu, ${ }^{1,2}$ \\ Carolyn J. Fisher, ${ }^{1,4}$ Philip R. Schauer, ${ }^{1,2}$ Alexander Rae-Grant, ${ }^{3,4}$ and Stacy A. Brethauer ${ }^{1,2}$ \\ ${ }^{1}$ Bariatric and Metabolic Institute, Cleveland Clinic, Cleveland, OH, USA \\ ${ }^{2}$ Department of General Surgery, Cleveland Clinic, Cleveland, OH, USA \\ ${ }^{3}$ Department of Neurology, Cleveland Clinic, Cleveland, OH, USA \\ ${ }^{4}$ Mellen Center for Multiple Sclerosis, Neurological Institute, Cleveland Clinic, Cleveland, OH, USA
}

Correspondence should be addressed to Kalman Bencsath; bencsak@ccf.org

Received 24 August 2016; Revised 13 January 2017; Accepted 1 February 2017; Published 19 February 2017

Academic Editor: Eliot Brinton

Copyright (C) 2017 Kalman Bencsath et al. This is an open access article distributed under the Creative Commons Attribution License, which permits unrestricted use, distribution, and reproduction in any medium, provided the original work is properly cited.

\begin{abstract}
Obesity is common in patients with multiple sclerosis (MS); however, safety and efficacy of bariatric surgery in this population remain unclear. A database of 2,918 was retrospectively reviewed, yielding 22 (0.75\%) severely obese patients with MS who underwent bariatric surgery. Sixteen surgical patients with complete follow-up data were matched to a nonsurgical control group of MS patients, based on age, BMI, MS subtype, and length of follow-up. MS relapse rates and trends in the timed twenty-five foot walk test (T25FW) were compared. In the surgical group (gastric bypass $n=19$, sleeve gastrectomy $n=3$ ), preoperative BMI was $46.5 \pm 7.2 \mathrm{Kg} / \mathrm{m}^{2}$ and average excess weight was $60.4 \mathrm{~kg}$. Follow-up data was collected at $59.0 \pm 29.8$ months. There were two major and four minor complications. Five patients required readmission and there were no mortalities. Percent excess weight loss was 75.5 $\pm 27.0 \%$. In the 16 patients with follow-up data, patients who underwent bariatric surgery were significantly faster on the T25FW compared to the nonsurgical population. In conclusion, bariatric surgery is relatively safe and effective in achieving weight loss in patients with MS. In addition, surgery may help patients maintain ambulation. Findings support the need for further studies on bariatric surgery and disease-specific outcomes in this population.
\end{abstract}

\section{Introduction}

Since 1999, obesity in the United States has been trending upwards, with the prevalence of obesity increasing from $27 \%$ to $35 \%$ in adult men and 33 to $36 \%$ in adult women [13]. Multiple sclerosis (MS) patients are not immune to the obesity pandemic. While the true rate of obesity in patients with MS is uncertain, estimates range from $21.2 \%-32.7 \%$ [4, 5]. Accumulating evidence suggests that adolescent obesity is a risk factor for developing MS, particularly in the context of certain genetic predispositions [6,7]. Moreover, MS commonly impairs walking ability, which can dramatically affect daily routines and quality of life [8], and for those patients with excess weight, it stands to reason that the disability from the disease may be even more pronounced. Despite the overlap between obesity and MS, literature pertaining to the safety and efficacy of bariatric surgery in the MS population is scarce. Therefore, we retrospectively reviewed our database and collected outcome data on bariatric surgery in this patient population. In addition, we matched these individuals to nonsurgical patients from our Neurologic Institute to compare walking outcomes between the two groups. To our knowledge, this is the first retrospective case-control series evaluating the long-term surgical and ambulatory outcomes of bariatric surgery in MS patients.

\section{Methods}

Approval for this project was obtained from our Institutional Review Board. A retrospective review of our bariatric surgical database from 2004 to 2012 was performed. A search by diagnosis and ICD-9 code corresponding to MS was used to 
identify the subset of individuals who carried the diagnosis of MS at the time of surgery. Demographic data, including age at the time of surgery, gender, preoperative weight and body mass index (BMI), and postoperative weight and BMI, were collected. Additional surgical outcomes analyzed included length of hospital stay, adverse events, readmissions, and mortality.

A control group of morbidly obese patients with MS who did not undergo bariatric surgery was identified through chart review of all MS patients presenting to our MS center during a consecutive 2-month period from February 1st to March 31st 2012. This group, within the limitations of a case-control series, was matched on BMI, age, MS subtype, and time interval between serial neurologic testing. The timed twenty-five foot walk test (T25FW), the European Quality of Life-5 Dimensions (EQ-5D), and the Patient Health Questionnaire (PHQ-9) were collected and analyzed whenever available. T25FW variability, defined as

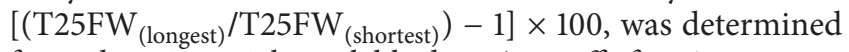
for each patient with available data. A cutoff of $20 \%$ or greater T25FW variability was considered reflective of significant clinical change, as validated in prior studies [9-11]. In addition, for each patient in either group, the preoperative (or initial) T25FW was subtracted from the postoperative (or most recent) T25FW (i.e., Post-Pre). This is designated herein as $\Delta \mathrm{T} 25 \mathrm{FW}$. Fisher's exact test and $t$-test were utilized to analyze the significance between groups. Data were analyzed using SPSS (Version 21, Chicago Inc., IL, USA) and a $p<0.05$ was deemed statistically significant.

\section{Results}

Review of the database yielded 2,918 patients who underwent bariatric procedures during the study period. Twenty-two of these patients $(0.75 \%)$ had a diagnosis of MS prior to bariatric surgery. Twenty patients were female and two were male with a mean age of $45.7 \pm 9.6$ years (range $32-62$ years). The mean BMI was $46.5 \pm 7.2 \mathrm{Kg} / \mathrm{m}^{2}$ (range $35.1-64.3 \mathrm{Kg} / \mathrm{m}^{2}$ ).

Nineteen patients underwent laparoscopic Roux-en-Y gastric bypass (RYGB) and three patients underwent laparoscopic sleeve gastrectomy. One of the patients previously had a laparoscopic adjustable gastric band placed and subsequently underwent conversion to a RYGB. The mean length of hospital stay was $4.2 \pm 4.4$ days (range 2-23 days). One patient required a hospital stay of 23 days due to complications of an anastomotic leak at the jejunojejunostomy. Five patients required early readmission: one patient was readmitted for dehydration, one for a port site infection, one for an anastomotic ulcer, and one for a stricture managed with balloon dilation. One patient required reoperation two weeks following RYGB for an adhesive obstruction at the distal ileum related to a prior hysterectomy.

At last follow-up (mean $=59.0 \pm 29.8$ months) the mean percentage of excess weight loss was $75.5 \% \pm 27.0$, and percentage of total weight loss was $31.5 \% \pm 11.8$.

No patients were on corticosteroids for management of MS at the time of surgery. Five patients were receiving daily injections of glatiramir, an immune modulator, just prior to surgery, with one of these patients also receiving interferon

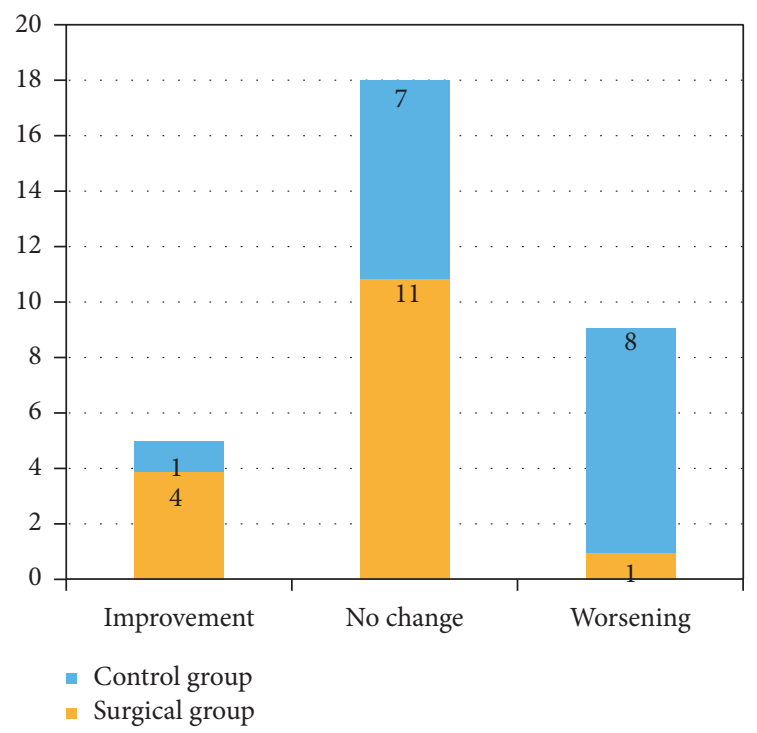

FIGURE 1: Distribution of T25FW variability across the two groups.

beta-1a three times weekly. Four other patients were also receiving interferon beta-1a. Two patients were receiving weekly injections of natalizumab, a humanized monoclonal antibody directed against the cell adhesion molecule alpha4integrin, for the treatment of MS.

The single patient with a port site infection was receiving interferon beta-la and the patient with an anastomotic ulcer was receiving glatiramir injections pre-operatively, but was also taking meloxicam until two weeks before surgery. The patient whose course was complicated by a jejunojejunal anastomotic leak was not receiving immune modulators for MS. Recent use of immune modulators for treatment for MS was not associated with complications of any kind $(p=1.00)$.

Pre- and postoperative T25FW data were available for 16 of the 22 patients who had bariatric surgery; four patients did not seek care at our institution for their MS and two additional patients lacked serial T25FW measurements. The baseline characteristics of these 16 patients and the nonsurgical control group are shown in Table 1. The mean time frame between the two index visits was 41.2 months for the study sample and 39.8 months for the control group. For the surgical group, 11 patients (68.7\%) showed no change in their walking speed, four patients (25\%) exhibited improvement, and one patient experienced slowing. This is in contrast with the findings among the 16 patients comprising the control sample, in which $8(50 \%)$ had slowing on serial testing, 7 (44\%) showed no change, and one patient exhibited improvement (Figure 1). After calculating the $\Delta \mathrm{T} 25 \mathrm{FW}$ for each group, it was noted that, on average, MS patients who underwent bariatric surgery were walking 1.96 seconds faster than their nonsurgical cohort ( $p=0.02$; Figure 2).

As for the quality of life measures, EQ-5D and PHQ-9 scores had improved on repeat assessment in both groups, with a nonsignificant difference between the two samples (data not shown). In addition, it is worthwhile to note that the number of acute MS exacerbations during the follow-up 
TABLE 1: Baseline characteristics of the surgical and control groups.

\begin{tabular}{lcc}
\hline & Surgical group $(n=16)$ & Control group $(n=16)$ \\
\hline Age, y, mean (SD) & $51(10.5)$ & $48.2(12.2)$ \\
Gender F-M & $15-1$ & $13-3$ \\
BMI, $\mathrm{kg} / \mathrm{m}^{2}$, mean (SD) & $45(4.7)$ & $43.6(3.7)$ \\
Type of MS & & 11 \\
$\quad$ Relapsing remitting (RRMS) & 2 & 15 \\
$\quad$ Secondary progressive (SPMS) & 3 & 0 \\
$\quad$ Primary progressive (PPMS) & & 2 \\
Medications & 1 & 0 \\
$\quad$ Dalfampridine ${ }^{\mathrm{a}}$ use & 0 & - \\
$\quad$ Corticosteroids & 5 & - \\
$\quad$ Glatiramir & 5 & - \\
$\quad$ Interferon beta-la & 2 & $9(4.3)$ \\
$\quad$ Natalizumab & $15.6(11.5)$ & $39.8(7)$ \\
Disease duration, years, mean (SD) & $41.2(28)$ & 4 \\
Length of follow-up, months, mean (SD) & 2 & \\
Number of acute MS exacerbations ${ }^{\mathrm{b}}$ & & \\
\hline
\end{tabular}

${ }^{\mathrm{a}}$ Indicated to help improve walking in adults with MS.

${ }^{\mathrm{b}}$ Exacerbations calculated over length of follow-up period noted in table.

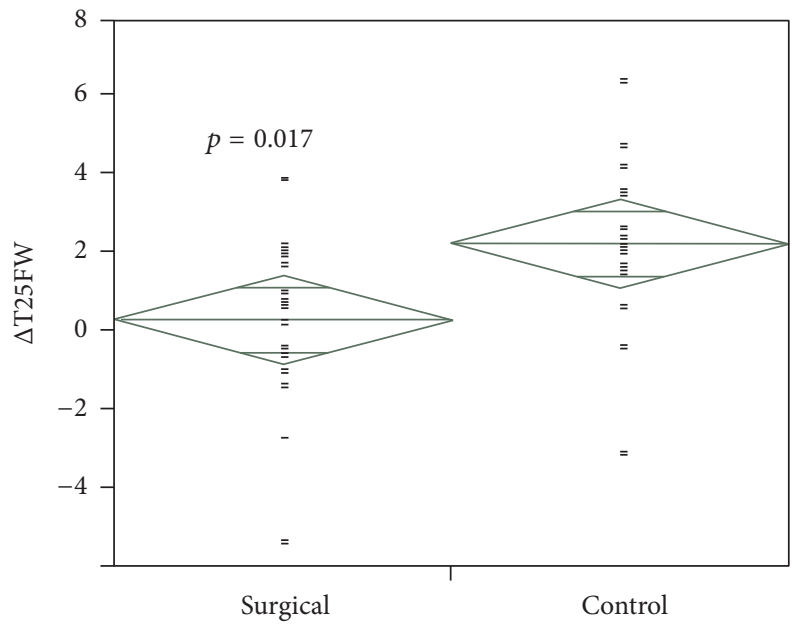

FIgURE 2: Distribution of (Post-Pre) T25FW differences between surgical and control groups.

period was 4 in the control group as compared to only 2 in the surgical group.

\section{Discussion}

In 1997, Flanagan reported the bariatric surgery outcomes in a heterogeneous case series of six patients with neurologic motor deficits, three of whom had MS. The mean excess weight loss was $77 \%$ at 18 months. Two of the MS patients demonstrated improved balance postoperatively while the third became wheelchair bound on long-term follow-up [12]. Lutrzykowski reported on a wheelchair-bound morbidly obese MS patient who experienced significant weight loss and improved mobility in her wheelchair after an open duodenal switch procedure [13].

Ambulation is frequently affected in MS patients, altering balance, impairing safety, and often slowing the speed of walking [14]. In many patients, ambulation is affected early in the disease, even when clinical measures of disability are minimally altered [15]. Of importance, patients with MS perceive difficulties with ambulation as a major issue for their health [16]. Ambulation limitations have an impact on activities, emotional status, quality of life, and health status [17]. A recent online survey revealed that two thirds of people with MS admitted to difficulty walking or maintaining balance, with most of them believing that that difficulty led to detrimental financial and social consequences such as unemployment [18].

No reports other than anecdotal data have been published on the surgical or ambulatory outcomes of bariatric surgery in MS patients. Our data are consistent with other studies reporting on bariatric surgery outcomes, particularly in higher risk populations, which have shown modest complication rates and a mean length of stay of 3-4 days [19, 20]. Long considered to be associated with an elevated risk of postoperative complications, the immune modulators used in this patient population do not seem to pose significant added risk. The readmission and overall complication rates are higher than typically seen and warrant further investigation and consideration of risk reduction measures in this patient population. A limitation of the study is the small sample size, however, and it is not known whether differences might be seen in larger samples. The average excess weight loss of $75.5 \%$ sustained by our study sample is on the upper end of the reported range of $57 \%$ to $77 \%$ seen in previous studies $[19,21]$.

With respect to MS outcome measures, our surgical patients were able to maintain stable walking speeds as 
measured by serial T25FW for the duration of follow-up, as compared to controls who were about 2 seconds slower on average. While this is an important finding, particularly in the context of limited research in this area, loss of excess weight is the most likely factor responsible for decreased walking time. This highlights a limitation of the current study, which was using this outcome measure in isolation. Future research should explore additional, well-accepted outcome measures of functional and disability status in this population following bariatric surgery.

The recent identification of childhood obesity as a risk factor for the development of MS raises the intriguing hypothesis that the metabolic effects of bariatric surgery may attenuate a unifying proinflammatory state underlying both obesity and MS [7, 22, 23]. Consistent with this idea, bariatric surgery has been found to have a significant positive effect on neurologic conditions such as pseudotumor cerebri, migraine, and Alzheimer's disease [24]. In this present study, there was no statistically significant difference in QOL measures between the two groups. The fact that worsening ambulation status in the control group did not translate into better QOL measures in the surgical cohort may be attributed to the small study sample size.

Due to growing evidence suggesting a link between obesity and MS, future research should seek to elucidate the nature of this relationship. Limitations of the current study pertain the cross-sectional and retrospective nature of the data analysis. In addition, while the control group was matched on relevant demographic and diagnostic variables, we do not have data on whether individuals in the control group were involved in nonsurgical weight management interventions, which may have attenuated our ability to detect differences in outcomes. Thus, a longitudinal controlled trial evaluating disease severity in patients with MS compared to MS patients undergoing bariatric surgery would help determine if bariatric surgery might be able to attenuate the disease and determine the mechanisms behind such a finding. Future studies should therefore be directed at disease-specific outcomes in bariatric surgery patients with MS.

Taken together, these data illustrate an overall acceptable safety profile of bariatric surgery in this patient population. In addition, we did not observe disease worsening or increased relapses following bariatric surgery. While the observational nature of this study precludes definitive conclusions, the results are encouraging. Our data suggest that bariatric surgery is a viable treatment option for the severely obese MS patient and that the surgical outcomes may be expected to be similar to the general bariatric population. The data is limited in the ability to confidently address the safety of immune modulators in the perioperative period, and this should be addressed on a case-by-case basis.

\section{Conclusions}

Bariatric surgery can be performed relatively safely and appears to be effective in patients with MS. The preoperative use of immune modulators for treatment of MS had no effect on perioperative complications in this small series.
These data support that surgery can be offered as part of a comprehensive strategy for the treatment of severe obesity in this patient population. Any positive impacts of surgery on the management of symptoms relating to MS, such as ambulation, or upon the disease itself should be explored in future studies.

\section{Competing Interests}

The authors declare that they have no conflict of interests.

\section{Acknowledgments}

The authors would like to thank Esteban Walker, Ph.D., for his invaluable assistance in statistical analysis and Stewart Tepper, M.D., and Richard Rudick, M.D., for their critical review of the initial proposal.

\section{References}

[1] C. L. Ogden, M. D. Carroll, B. K. Kit, and K. M. Flegal, "Prevalence of childhood and adult obesity in the United States, 2011-2012," Journal of the American Medical Association, vol. 311, no. 8, pp. 806-814, 2014.

[2] M. L. Baskin, J. Ard, F. Franklin, and D. B. Allison, "Prevalence of obesity in the United States," Obesity Reviews, vol. 6, no. 1, pp. 5-7, 2005.

[3] K. M. Flegal, M. D. Carroll, B. K. Kit, and C. L. Ogden, "Prevalence of obesity and trends in the distribution of body mass index among US adults, 1999-2010," The Journal of the American Medical Association, vol. 307, no. 5, pp. 491-497, 2012.

[4] S. R. Khurana, A. M. Bamer, A. P. Turner et al., "The prevalence of overweight and obesity in veterans with multiple sclerosis," American Journal of Physical Medicine and Rehabilitation, vol. 88, no. 2, pp. 83-91, 2009.

[5] L. A. Pilutti, D. Dlugonski, J. H. Pula, and R. W. Motl, "Weight status in persons with multiple sclerosis: implications for mobility outcomes," Journal of Obesity, vol. 2012, Article ID 868256, 6 pages, 2012.

[6] A. K. Hedström, T. Olsson, and L. Alfredsson, "High body mass index before age 20 is associated with increased risk for multiple sclerosis in both men and women," Multiple Sclerosis Journal, vol. 18, no. 9, pp. 1334-1336, 2012.

[7] A. K. Hedström, I. L. Bomfim, L. Barcellos et al., "Interaction between adolescent obesity and HLA risk genes in the etiology of multiple sclerosis," Neurology, vol. 82, no. 10, pp. 865-872, 2014.

[8] R. W. Motl, "Ambulation and multiple sclerosis," Physical Medicine and Rehabilitation Clinics of North America, vol. 24, no. 2, pp. 325-336, 2013.

[9] M. Kaufman, D. Moyer, and J. Norton, “The significant change for the Timed 25-Foot Walk in the multiple sclerosis functional composite," Multiple Sclerosis, vol. 6, no. 4, pp. 286-290, 2000.

[10] C. I. Coleman, D. M. Sobieraj, and L. N. Marinucci, "Minimally important clinical difference of the Timed 25-foot walk test: results from a randomized controlled trial in patients with multiple sclerosis," Current Medical Research and Opinion, vol. 28, no. 1, pp. 49-56, 2012.

[11] B. C. Kieseier and C. Pozzilli, "Assessing walking disability in multiple sclerosis," Multiple Sclerosis Journal, vol. 18, no. 7, pp. 914-924, 2012. 
[12] L. Flanagan Jr., "Is bariatric surgery effective in the treatment of the neurological motor deficit syndromes?" Obesity Surgery, vol. 7, no. 5, pp. 420-423, 1997.

[13] M. Lutrzykowski, "Bariatric surgery in morbidly obese patients in wheelchairs," Obesity Surgery, vol. 18, no. 12, pp. 1647-1648, 2008.

[14] R. J. Swingler and D. A. Compston, "The morbidity of multiple sclerosis," Quarterly Journal of Medicine, vol. 83, no. 300, pp. 325-337, 1992.

[15] C. L. Martin, B. A. Phillips, T. J. Kilpatrick et al., "Gait and balance impairment in early multiple sclerosis in the absence of clinical disability," Multiple Sclerosis, vol. 12, no. 5, pp. 620-628, 2006.

[16] C. Heesen, J. Böhm, C. Reich, J. Kasper, M. Goebel, and S. M. Gold, "Patient perception of bodily functions in multiple sclerosis: gait and visual function are the most valuable," Multiple Sclerosis, vol. 14, no. 7, pp. 988-991, 2008.

[17] H. L. Zwibel, "Contribution of impaired mobility and general symptoms to the burden of multiple sclerosis," Advances in Therapy, vol. 26, no. 12, pp. 1043-1057, 2009.

[18] Harris Interactive, Survey Finds Majority of People with Multiple Sclerosis Report Difficulty Walking or Maintaining Balance, National MS Society and Acorda Therapeutics, New York, NY, USA, 2011.

[19] P. R. Schauer, S. Ikramuddin, W. Gourash, R. Ramanathan, and J. Luketich, "Outcomes after laparoscopic Roux-en-Y gastric bypass for morbid obesity," Annals of Surgery, vol. 232, no. 4, pp. 515-529, 2000.

[20] A. Aminian, M. H. Jamal, A. Andalib et al., "Is laparoscopic bariatric surgery a safe option in extremely high-risk morbidly obese patients?" Journal of Laparoendoscopic and Advanced Surgical Techniques, vol. 25, no. 9, pp. 707-711, 2015.

[21] H. Buchwald, Y. Avidor, E. Braunwald et al., "Bariatric surgery: a systematic review and meta-analysis," Journal of the American Medical Association, vol. 292, no. 14, pp. 1724-1737, 2004.

[22] A. Langer-Gould, S. M. Brara, B. E. Beaber, and C. Koebnick, "Childhood obesity and risk of pediatric multiple sclerosis and clinically isolated syndrome," Neurology, vol. 80, no. 6, pp. 548552, 2013.

[23] K. L. Munger, T. Chitnis, and A. Ascherio, "Body size and risk of MS in two cohorts of US women," Neurology, vol. 73, no. 19, pp. 1543-1550, 2009.

[24] S. A. Brethauer, B. Chand, and P. R. Schauer, "Risks and benefits of bariatric surgery: current evidence," Cleveland Clinic Journal of Medicine, vol. 73, no. 11, pp. 993-1007, 2006. 


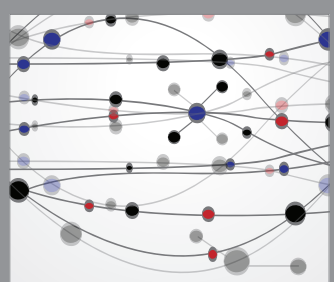

The Scientific World Journal
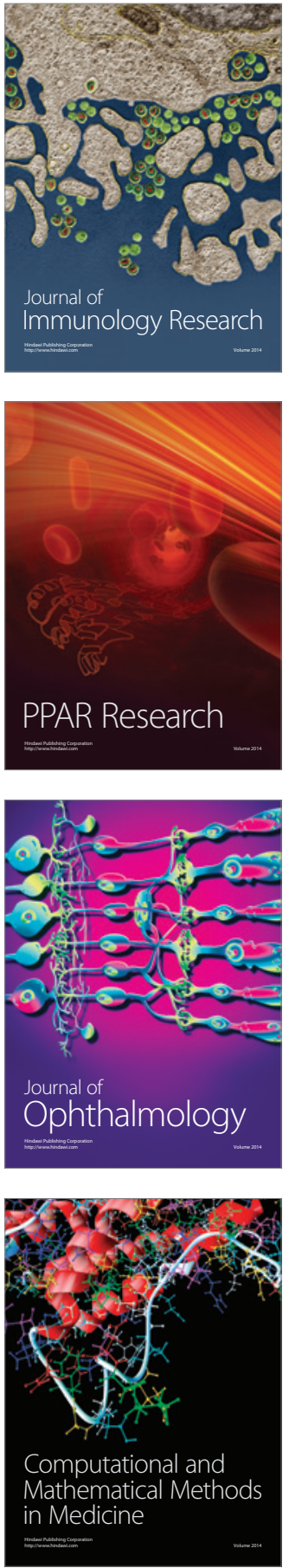

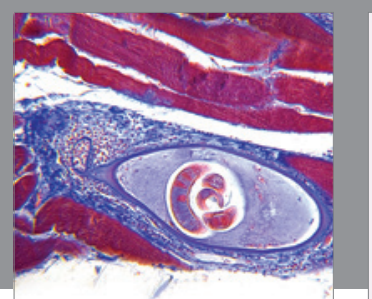

Gastroenterology Research and Practice
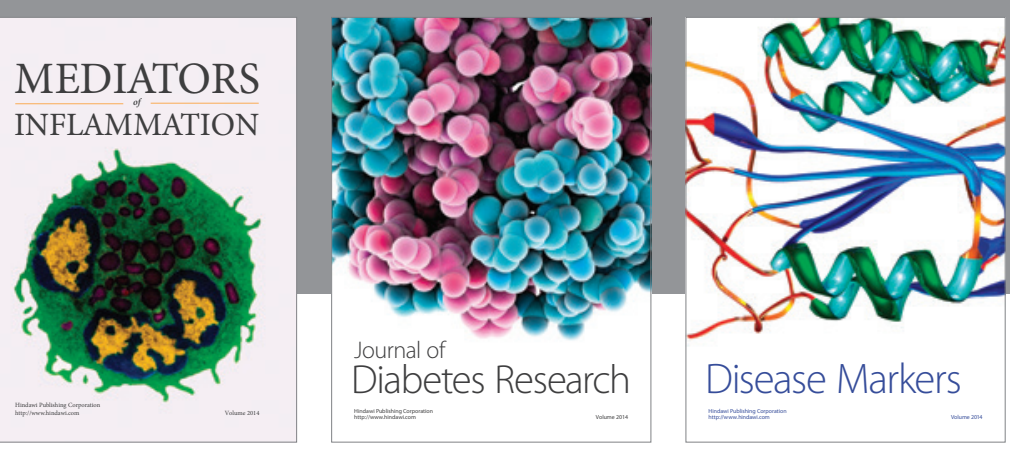

Disease Markers

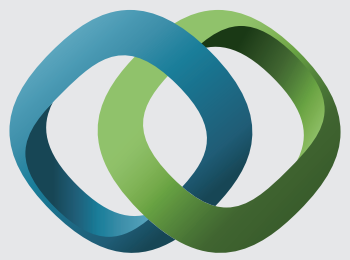

\section{Hindawi}

Submit your manuscripts at

https://www.hindawi.com
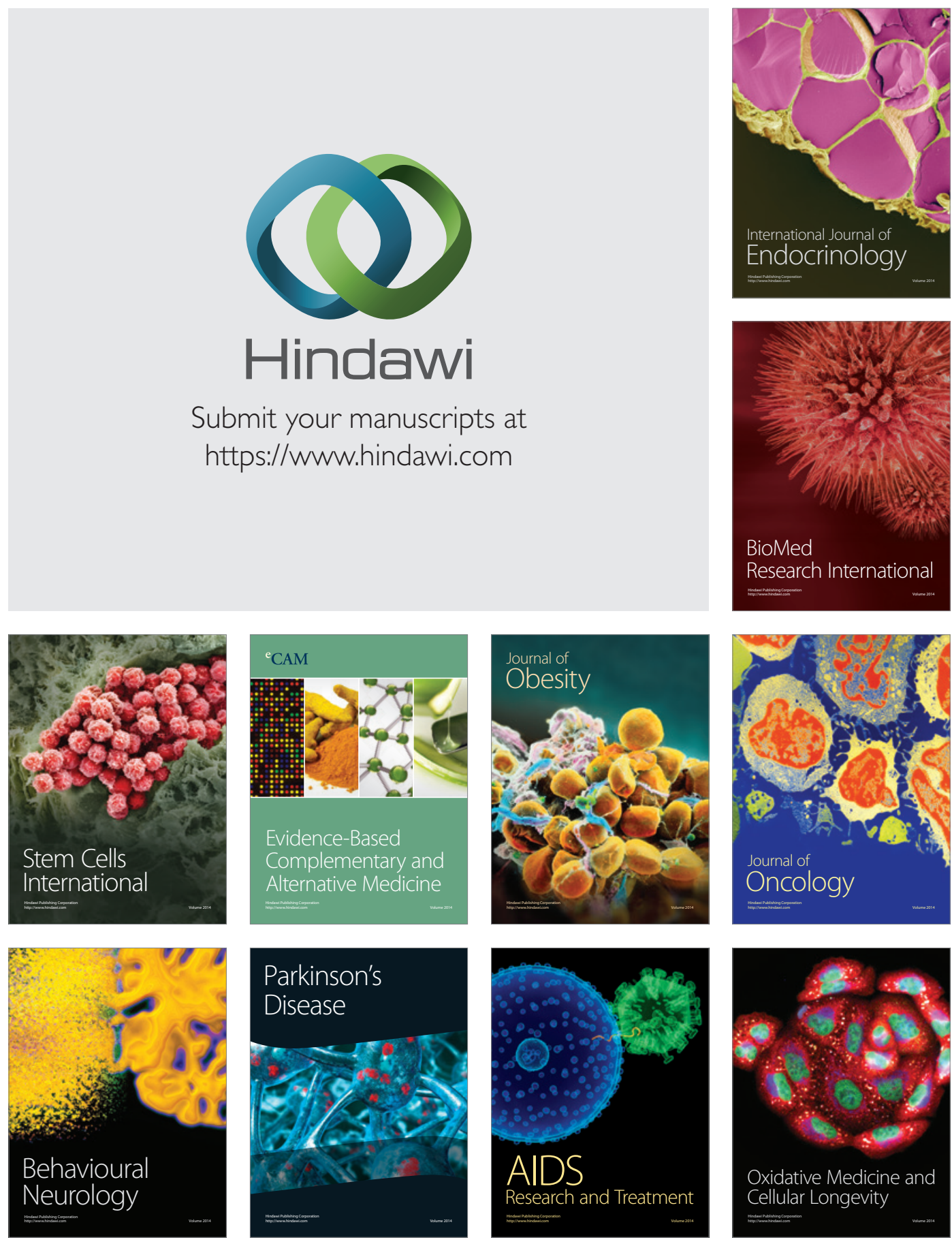